\title{
Inhaltsverzeichnis.
}

\author{
Viertes Buch. \\ Dau Zeitalter der Aufklärung. Allmähliches $\Delta$ ufsteigen \\ des Neuhumanismus. 1740-1805.
}

Erstes Kapitel.

Der allgemeine Cbarakter des Zeitalters und sein Verhältnis zum Altertum . . . . . . . . . . . . . . . .

Die Aufklärung als Reaktion gegen den Pietismus (3). KLOPSTOCK und WrnckelmanN, poetische und ästhetische Sensibilität (5). Nationale Empfindung (6). Wiederbelebung des Homer (7).

\section{Zweites Kapitel.}

Die neue Universität Göttingen. Neuhumanistische Philologie und Gymnasialpädagogik; GrSner, Ernestr, Heyne, Herdar

Charakter der neuen Universität Göttingen (9). Bestand und Ausstattung (12). Vorlesungen (14). Entstehung des neuen Humaniamus (15). Joh. Matthisas Grsnbr (16); s eine Gymnasialpädagogik: Gegensatz althumanistischer und neuhumanistischer Anschauung (17). Seine Ansichten über Ziel und Methoden des klassischen Unterrichts (18), über Art und Nutzen der Lektüre (23). GESNER in Leipzig und Göttingen (24). Das Göttingische philologische Seminar (25). Förderung der griechischen Studien (26). Seine Vorlesungen (29). JоH. AJG. ERNESTI in Loipzig; seine gymnasialpädagogischen Ansichten (30); kursächsische Schulordnung (32); Schüler (33). J. G. Reiske (33). DnNTrars Bericht über seine Schulzeit in Grimma (34). - Chr. G. HEYNE: allgemeine Bedeutung (36). Seine akademische Lehrtätigkeit (38). Organisation der Schule zu Ilfeld (40). Schüler (42). - HrRDers Reform des Weimarer Gymnasiums (43). Philologisch-pädagogische Seminare in Helmstedt und Erlangen (46).

\section{Drittes Kapitel.}

IPlilanthropische Pädagogik. Anfänge der Real-oder Bürger. schule . . . . . . . . . . . . . . . . . . .

Rousseaus Emil, Tendenz und Wirkung (48). Verhältnis der Philanthropinisten zu den Humanisten (50). J. B. BASEDOw (51). Das Elementarwerk und die Begründung des Philanthropins (52). Die Ankündigungsschrift (52). Der Lehrgang des Philanthropins (56). - E. Cr. Trapp: seine Ansichten über Gymnasialpädagogik und Schulreform (57). Stimmen 


\section{Viertes Kapitel.}

Der Neubau der Gelehrtenschule auf neuhumanistischer Grund lage in PreuBen (1808-1818) . . . . . . . . . . . 278

Die Wiederaufrichtung des preußischen Staates und die neue Erziehung (278). Pestalozzi, Fichte, Freiherr v. Strin (279). Bedeutung des Humanismus für das neue Erziehungsideal (281.) Die Organisation des Gelehrtenschulwesens durch W. v. HumboLDT, SüvERN, Nicolovrus (282): 1. Die Ausbildung der staatlichen Schulverwaltung (283). 2. Die Schaffung eines eigenen Gymnasiallehrerstandes; Lehramtsprüfung (286). 3. Die Fixierung des Gymnasialkursus. Abiturientenprüfung (288). 4. Der Lehrplan des Gymnasiums. Süvmans Unterrichtsverfassung (290). F. A. Wourrs Kritik (296). SüvkRNs Entwurf eines Unterrichtsgesetzes und die Folgen seines Scheiterns (299).

Beispiele der Ausführung der neuhumanistischen Reformideen: Poppo in Frankfurt a. 0. (302). MerNeke in Danzig (305). SpItzar Er in Wittenberg (307). GotrmoLD in Königsberg (308). Rückblick auf den neuhumanistischen Umschwung und seine Ursachen (309).

\section{Fünftes Kapitel.}

Der Ausbau des neuen Gymnasiums in Preußen. Johannes

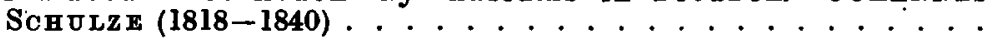

Friedrich Wilhelm III. und seine Regierung (316). Der Minister voN Altensteit (317). Johannes Schulze: Bildungsgang und Persönlichkeit (318). Charakter aeiner Wirksamkeit (322). Einfluß der Demagogenverfolgungen (323). Verordnungen ScHULzes: Latein (327), Griechisch (328), Prïvatlektüre (329), deutsche Lektuire (330), Philosophie (330). Klassenlehrersystem (333).

Beginnender Widerspruch gegen ScHoLze. Uberbürdungsklagen: Fr. Thimgsch (336). F. v. Raumer (336). Widerstand der Provinzialschulkollegien (337). Die Utberbürdung (341). Einlenken ScHULzas (344). Lehrerprüfungsreglement von 1831; Abiturientenprüfungsordnung von 1834 (347). Lorinsers Angriff (349). Das ,blaue Buch" und der Normalplan von 1837 (351).

Die Schulreform in Hannover (356), in Braunschweig (358), in Schleswig-Holstein und Lübeck (359), in Hessen (360).

\section{Sechstes Kapitel.}

Das Urteil über das neue preußische Gymnasium . . . . . . . .

J. Schulzks Verdienste um das Bildungswesen (382). Bedenkliche Seiten der Reglementierung (364). Ungünstige Urteile über das neue Gymnasium: SPIllekr (369). G. KöPKE, M. Schmmt (371). ScharberT (372). Provinzialschulräte Jachmann, Otтo SchUlz (376). Ellers, Herbart (377). - Urteile über die Leistungen in den alten Sprachen: K. Lachmany, K. Mager (379). M. Seytert, Inghreslev (380). H. Thirrsoh, Th. WatTz (381). Urteile von Männern außerhalb der Schulwelt: Froritep (382). Goterie, H. Stefrews, Al. v. Humboldt (383). Schülererinnerungen: G. Parthey (385). E. v. Richithofen (386). Urteil P. de Lagardes (388).

Der Gymnasiallehrerstand: steigendes Ansehen (389). Neue Stellung zur Gesellschaft (390). Höhere wissenschaftliche Ausbildung (391). Wirksamkeit -(392). Schulaufsicht (395). Rückwirkung auf das Uni- 
versitätsstudium (396). - Wandlung des Unterrichtsverfahrens (399). Das Präparationssystem (401). Das Lateingchreiben (403). Endergebnis (405).

\section{Siebentes Kapitel.}

Die neuhumanistische Reform der Gelehrtenschule in den mittelund süddeu tschen Staaten ...............406

GotTfrimd Hermans als Repräsentant der älteren Form des Neúhumanismus (407). Fr. THIEzSCH (410). Die sächsischen Fürstenschulen im Anfang des 19. Jahrhunderts (410). Die neue Entwicklung (416). Sächsischer Lehrplan von 1846 (418).

Die neuhumanistische Schulreform in Bayern (421). NiETHAmmirs Organisationsentwurf vom Jahre 1808 (422). Ludwig I. und FrIEDRICH ThIERSCH (423). Universität München. Thrmeschs philologi ches Seminar (424). THIERSOHs Gymnasialpädagogik und der Schulplan vom Jahre 1829,30 (425). Widerspruch gegen denselben (430). Reaktion in Bayern (431). THImpschs weitere Wirksamkeit (434). Genossen des Werkes (434).

Die württembergischen Schulen in diesem Zeitalter: Klosterreform von $1806 / 7$ (435). Universitätsreform von 1811 (436). Zustand der Gymnasien und Lateinschulen (438). Der neue Humanismus in Baden (441).

\section{Sechstes Buch.}

Strebnngen und Gegenstrebungen in der Zeit von 1840-1892.

Übersicht . . . . . . . . . . . . . . . . . 44

\section{Erstes Kapitel.}

Die allgemeinen Tendenzen des neuen Zeitalters . . . . . . . 447

Veränderungen in der politischen, kirchlichen, wissenschaftlichen Welt (447), auf dem Gebiete der philologisch historischen Studien (449). Veränderte Stellung der klassischen Philologie (450). Fr. RITSCHL (451). Rückwirkung auf den Schulunterricht (453).

\section{Zweites Kapitel.}

Friedrich Wilhelm IV. und seine Gymnasialpolitik. ErchHorN und Gerd EILERS $(1840-1848)$. . . . . . . . . . 456

Friedrich Wilhelms Anschauungen und Absichten (456). Ministerialverfügung vom 10. Januar 1848 (459). Ministerium EichHORN (461). G. EILERS und seine Kritik am Universitäts- und Schulwesen (462). Sein Krieg mit den ,heidnischen“"Philologen (464). Verordnungen und Versuche persönlicher Einwirkung (470). Persönlichkeit Friedrich Wilhelms IV. (472).

\section{Drittes Kapitel.}

Døs Revolutionsjahr 1848 und die österreichische Gymnasialreform .................. . . 473

Die Lehrerversammlungen, Abneigung gegen den Lateinzwang (473). H. KöchlY (474). K. v. RAUMER und Rektor ElsPergar (475). Landesschulkonferenz und Schulgesetzentwurf des Ministeriums LaDENDorF (477). 
Das österneichische Gymnasialwesen vor 1848 (479). Ministerium THUN; EXnkr und Bonrtz (481). Der Organisationsentwarf von 1849 (482). Vergeblicher Widerstand gegen denselben (486). - Die bayerischen Schulpläne von 1854 und 1873 (489). Württembergische Schulreform (490).

\section{Viertes Kapitel.}

Das preuBische Gymnasialwesen im Zeitalter der Reaktion. LODW IG WIRSE (1850-1866) . . . . . . . . . . . . . 481

Minister v. RAUMFR und seine Schulpolitik (491). LODW. WIESE (494). Die konfessionelle Bewegung und die Erlanger Philologenversammlung (495). Stellung der Gymnasialwelt zu dem Gegensatz von Christentum und Altertum (498). Wreses gymnasialpädagogische Anschauungen (502). M. SEYFFrerT ,Uber das Privatstudium" (504). MaBregeln zur Erhaltung des christlichen Charakters der Gymnasien und Erfolg derselben (507). - Revision des Lehrplans. Konzentrationsbestrebungen in Richtung auf die alte Lateinschule (512). Lehrplan und Prüfungsordnung von 1856 (517). Mißerfolge (520). WIFSEs Stellungnahme dazu (525); andere Stimmen: Schradme (529). LaNDFRrmanN (530). Negatives Ergebnis: Der Lateinunterricht (532). Die Universitäten geben das Latein auf (536). Der griechische Unterricht: LEHRS und SchniBent darüber (538). Die Vorbildung der Gymnasiallehrer (540). Gesamturteil (542).

\section{Fünftes Kapitel.}

Die Entwicklung der Realschule zum Gymnasium in Preußen

Rückblick auf die frühere Entwicklung (544). Jor. Schulare und die Realschule (546). Aufnahme des Lateinischen (550). Der Gedanke eines modern gerichteten Gymnasiums (551). Ordnung der Entlassungsprüfung von 1832 (553). v. HTPPELs Vorschlag (555). Weitere Entwicklung in der Revolutions- und der Reaktionszeit (555). Unterrichtsund Prüfungscrdnung von 1859. Die Realschule erster Ordnung (558). Der Kampf um die Zulassung der Realschule zur Universität (564). Beschränkte Zulassung seit 1870 (567). Die FaIJ sche Schulkonferenz von 1872 (568). BonITz und die Lehrpläne vom Jahre 1882: Realgymnasium; Aufsteigen der lateinlosen Realschule (570).

Entwicklung des technischen Hochschulwesens (574).

\section{Sechstes Kapitel.}

Die Lehrpläne von 1882 und 1891 . . . . . . . . . . .

Die neue Ära: Minister v. BhTHMann-HoLlwgG (576). Ministerium Fatr (577). Herm. Bontrz und die Lehrpläne von 1882 (560). Erfolglosigkeit auch dieser Reform. Ưberbürdung der Schüler (584). Der Kampf um das Gymnasialmonopol (588). Entwicklung der lateinlosen Realschulen (591). Schulreformvereine (593). Die Schulkonferenz von 1890 (595). Ihre Beschlüsse (599). Die Lehrpläne von 1891 (601). Das Realgymnasium (605); die lateinlose Realschule (606). Die Prüfungen (606). Kritik der Ergebnisse (608). Die Abschlußprüfung (611). Bedeutung und Wert der neuen Schulverfassung: das Gymnasium (614): Begünstigung der lateinlosen Anstalten (616). Die Behandlung der Realgymmasien (617). Die Notwendigkeit des Realgymnasiums (618). 
Die ReformanstaIten nach dem Altonaer und Frankfurter System (622). Rückblick auf den Bildungsgang des Verfassers (625). AbschluB (626). Die Lehrerbildung: die Prüfungsordnung vom Jahre 1887. Die Gymnasialseminare (628). Das Probejahr (630). Beurteilung der Verändérungen (630). Die Entwricklung in den übrigen deutachen Stasten (633); in Osterreich (635).

\section{SchluBbetrachtung.}

Die Zukunft des gelehrten Unterrichts . . . . . . . . . . .637

Persönliche Stellung des Verfassers zum Altertum und zum Gymnasium (637). Veränderte Bedeutung des Altertums für das Leben (638). Folgerungen für die Schule (640). Der Utraquismus und seine Folgen (642). Die erwarteten und die tatsächlichen Fríchto des klassischen Unterrichts (649). Humane Bildung und klassischer Unterricht (653). Andere Wege zur humanistischen Bildung (661). Übersetzungen und ihr Wert (665). Der deutsche Unterricht (667). Der philosophische Unterricht (670). Ungerechtfertigte Bedenken gegen diese Fächer (674). GröBere Selbständigkeit und Individualisierung ist anzustreben, (681). - Das Verhältnis der gelehrten Bildung zur Volksbildung. Sozialaristokratiomus (682). Das Ideal der ,allgemeinen Bildung“" (689). Die Zukunft des Griechischen (692).

\section{Anhang.}

\section{Der gelehrte Onterricht bis anm Weltkrieg. 1892-1914.}

\section{Erstes Kapitel.}

Die Universitäten am Ende des 19. und zu Beginn des 20. Jahrhunderts . . . . . . . . . . . . . . . . . . . 695

Neue Universitäten (695). Ansteigen der Hörer- und Lehrerzahlen; Statistisches darüber (696). Wandel in der Stellung zum nationalen Leben (697). Aufschwung der technischen und Fachhoohschulen (698). Popularisierung der Wissenschaft (699). Das Selbstverwaltungsrecht und der Staat (700). Friedrich Althoff, seine Persönlichkeit (701) ; seine Universitätsverwaltung (704). Die Nichtordinarien-Bewegung (707). Schwierigkeiten der Weiterentwicklung (709). - Die philosophischen Fakultäten: fortschreitende Spezialisierung. Zurücktreten der Philosophie (710). Die Vorbildung für das höhere Lehramt (712); Vernachlässigung der Erziehungswissenschaft (714).

\section{Zweites Kapitel.}

Die Reform der höheren Schulen zu Beginn des 20. Jahrhunderts 715

Unbefriedigendes Ergebnis der Neuordnung vom Jahre 1892 (715). Arbeit für die Weiterentwicklung der realistischen und modernen Lehrfächer: Physik (718), neuere Sprachen (719) das Deutsche (721). Der Kampf um die Gleichberechtigung (722). Bedeutung der Reformanstalten (723). Soziale Bedeutung des Schulstreits (725). P. Ca derr Forderuing der Gleichberechtigung (728). Friedrich Padlsens Persönlichkeit und Bedeutung (729). Schwankende Stellung der Schul- 
verwaltung. Eingreifen Althorrs (732). Vorbereitung der Junikonferenz (734). Kundgebungen aus den Fachkreisen (735). Die Schulkonferenz im Juni 1900 (736): die Gleichberechtigungsfrage (737); der gemeinsame Unterbau (739); der griechische Unterricht (741); sonstige. Beschlüsse (744). Gesamtcharakter der Konferenz (745). Die Gleichberechtigung durch den Kaiserl. Erlaß vom 26. November ausgesprochen; ihre weitere Durchführung (746). Die neuen Lehrpläne (748); die Bestimmungen für die klassischen Sprachen (749). Allgemeiner Charakter der Lehrpläne und Gesamtergebnis der Schulreform (754). Weiterentwicklung der Reformgedanken: A. MatTmas' Monatsschrift(758). Die Reformanstalten (759). Die İdee der „Bewegungsfreiheit“, Pläne und Versuche zu ihrer Verwirklichung (761). Verschiebungen im Verhältmis der realen und gymnasialen Anstalten (764). Ende der Reformarbeit in Preußen (767).

Die Reform in den kleineren Staaten (767); Neuordnung in Württemberg (768), in Bayern (769). Die österreichische Schulreform (772).

Die Reform des höheren Mädchenschulwesens (774). Die Reformbewegung seit 1872 (775). Die Haltung der preußischen Unterrichtsverwaltung. Sr. WaETzor.dT (777). Die Konferenz im Jahre 1904 und die Neuordnung vom Jahre 1908 (778). Entsprechende Regelung in den übrigen deutschen Staaten (781).

Ausblick auf die künftige Gestaltung der höheren Schule. Die Stellung der Schulverwaltung (782). Die Eigenart der vorhandenen Schultypen und ihre Erhaltung (784). Die nationale Schule (786).

Anti-intellektualistische Strömungen im Schulwesen (787). Entwicklung der Sports (788). Wandervogel und Freideutsche Jugendbewegung (789). Die individualistische Richtung (790). Landerziehungsheime (792). Arbeitsschule und staatsbürgerliche Erziehung (792).

Schluß: der gelehrte Unterricht und der Einheitsschulgedanke (795).

Vollständige Titel einiger öfter verkürztangeführten Schriften

Register:

I. Namenregister . . . . . . . . . . . . . . . 806

II. Ortsregister . . . . . . . . . . . . . . . 821

III. Sachregister. . . . . . . . . . . . . . . . . 826 